\title{
Sympathetic cooling in two-species ion crystals in a Penning trap
}

\author{
S. Schmidtt ${ }^{\mathrm{a}, \mathrm{b}}$, T. Murböck ${ }^{\mathrm{c}}$, Z. Andelkovic ${ }^{\mathrm{d}}$, G. Birkl ${ }^{\mathrm{c}}$, K. König ${ }^{\mathrm{a}}$, W. \\ Nörtershäuser ${ }^{\mathrm{a}}$, R.C. Thompson ${ }^{\mathrm{e}}$ and M. Vogel ${ }^{\mathrm{d}, \mathrm{f}}$ \\ anstitut für Kernphysik, Technische Universität Darmstadt, 64289 Darmstadt, Germany; \\ bInstitut für Kernchemie, Universität Mainz, 55099 Mainz, Germany; \\ 'Institut für Angewandte Physik, Technische Universität Darmstadt, 64289 Darmstadt, \\ Germany; \\ ${ }^{\mathrm{d}}$ GSI Helmholtzzentrum für Schwerionenforschung, 64291 Darmstadt, Germany; \\ e Department of Physics, Imperial College London, SW7 2AZ London, UK; \\ ${ }^{\mathrm{f}}$ Helmholtz-Institut Jena, 07743 Jena, Germany
}

\section{ARTICLE HISTORY}

Compiled June 22, 2017

\begin{abstract}
We have studied the formation and properties of two-species ion Coulomb crystals in the Penning trap of the SpecTrap experiment. These crystals have been formed by injection of admixture ions from an external source into a previously confined and laser-cooled cloud of magnesium ions. This kind of study, performed over a range of the admixture ions' charge-to-mass ratios, indicates the conditions for their sympathetic cooling and the formation of two-species ion crystals. This mechanism allows efficient cooling of the admixed species such as highly charged ions which do not feature suitable laser-cooling transitions, and thus make them accessible to high-resolution laser spectroscopy.
\end{abstract}

\section{KEYWORDS}

laser cooling; Penning trap; ion Coulomb crystal; laser spectroscopy

\section{Introduction}

Precision spectroscopy of optical transitions in atoms and ions requires efficient cooling of their motion to minimize line shifts and broadening due to the Doppler effect (1). For atomic and molecular ions confined in Penning traps (2, 3), several cooling techniques have been developed (4), partly allowing ions to be cooled down to the quantum mechanical ground state of their motion in the trap (5). The most common techniques applied in Penning traps are buffer gas cooling $(6)$, resistive cooling $(7,8)$ and various forms of laser cooling $(5,9,10)$. Buffer gas cooling and resistive cooling are universal in the sense that they are independent of the ions' internal properties, but usually limited to the ambient temperature which often is liquid helium temperature of around $4 \mathrm{~K}$. Laser cooling goes much below that. Typical limits for laser Doppler cooling are in the $\mathrm{mK}$ regime and more elaborate techniques allow even lower temperatures (10-12). On the other hand, laser cooling relies on the presence of suitable optical transitions that can be driven by lasers. In many ion species, including highly charged ions, suitable transitions do not exist or are inaccessible by present-day lasers (13). However, when laser-cooled ions are confined together with such species, they provide cooling mediated 
by the Coulomb interaction, named 'sympathetic cooling' (4, 14).

We have studied the sympathetic cooling of a number of different admixture ion species injected into a Penning trap filled with laser-cooled magnesium ions with $m / q=24 \mathrm{u} / \mathrm{e}$ for ${ }^{24} \mathrm{Mg}^{+}$. Under the present conditions, the magnesium ions form near-planar crystalline structures (15), and the second species can become embedded in the crystal, depending on the confinement details. Measurements have been performed for mesoscopic magnesium ion crystals injected with singly charged ions of mass-to-charge ratios $m / q=2 \mathrm{u} / \mathrm{e}\left(\mathrm{H}_{2}^{+}\right), m / q=12 \mathrm{u} / \mathrm{e}\left(\mathrm{C}^{+}\right), m / q=28 \mathrm{u} / \mathrm{e}\left(\mathrm{N}_{2}^{+}\right)$, and $m / q=44 \mathrm{u} / \mathrm{e}\left(\mathrm{CO}_{2}^{+}\right)$.

\section{Ion Coulomb crystals in a Penning trap}

A Penning trap provides confinement of charged particles such as ions by a superposition of a static homogeneous magnetic field along the trap's central axis with a static electric quadrupole potential $(2,3)$. The former provides radial trapping on account of the Lorentz force, while the latter confines the ions in a harmonic potential well along the axis. Under ideal trapping conditions, i.e. for perfect field geometry, an ion performs a motion that is the superposition of three independent oscillatory motions, two in the radial plane (perturbed cyclotron and magnetron motions) and one in the axial direction (16). The frequencies of these motions are labelled $\omega_{+}, \omega_{-}$and $\omega_{z}$, respectively. They typically obey a hierarchy with $\omega_{+} \gg \omega_{z} \gg \omega_{-}$. For a ${ }^{24} \mathrm{Mg}^{+}$ion in the present trap at a trapping voltage of $U=50 \mathrm{~V}$ and a magnetic field strength of $B=4.1 \mathrm{~T}$, these frequencies are $\omega_{z}=2 \pi \times 241.4 \mathrm{kHz}, \omega_{-}=2 \pi \times 11.4 \mathrm{kHz}$ and $\omega_{+}=2 \pi \times 2.55 \mathrm{MHz}$.

For a single ion, the amplitudes of these motions are independent from one another. In case of the cyclotron and axial motions, a reduction of the ion kinetic energy by any means of cooling will reduce the respective oscillation amplitude (16). In the present experiment, we are concerned mainly with the axial direction since the laser cooling is performed along the trap's main axis. The axial oscillation amplitude $a_{z}$ of an ion is related to its axial kinetic energy $E_{z}$ by

$$
a_{z}^{2}=\frac{2 E_{z}}{m \omega_{z}^{2}},
$$

which under the present condition leads to an amplitude of roughly one $\mu \mathrm{m}$ at an ion energy of one $\mu \mathrm{eV}$, corresponding to a temperature of about $10 \mathrm{mK}$.

In confined ion ensembles (which are one form of a non-neutral plasma) the motions become coupled due to ion-ion interaction via the Coulomb force. Again, a reduction of the ion kinetic energy (the ensemble temperature $T$ ) will lead to reduced amplitudes of the cyclotron and axial motions, but also to a decrease in ion-ion distance which in a cold plasma leads to a strong correlation between the ions that is described by the plasma parameter

$$
\Gamma_{p}=\frac{q^{2}}{4 \pi \epsilon_{0} a_{w s} k_{B} T},
$$

where $\epsilon_{0}$ is the permittivity of free space and $k_{B}$ is the Boltzmann constant. The Wigner-Seitz radius $a_{w s}$ (the average ion-ion distance) is defined by $a_{w s}^{3}=3 /(4 \pi n)$ at the ion number density $n$. The collective behaviour of confined ions in terms of a 
non-neutral plasma has been described in detail for example in (17).

At sufficiently low temperatures $T$ (i.e. for sufficiently large plasma parameter $\Gamma_{p}$ ), the ions form ordered structures determined by the balance of their mutual Coulomb repulsion and the common confining potential. These configurations are referred to as 'ion Coulomb crystals' (18), and have been observed in numerous experiments and for a broad range of experimental conditions (19-26). In a Penning trap, due to the presence of the magnetic field used for ion confinement, the crystals perform a global rotation about the trap's central axis. In the absence of external torques for example by radial lasers, buffer gas or rotating wall drives $(27)$, the rotation frequency remains constant and its value depends mainly on the initial conditions (28). For externally produced ions, these initial conditions are largely given by the details of ion capture. The global rotation frequency $\omega_{r}$ lies between the magnetron frequency $\omega_{-}$and the reduced cyclotron frequency $\omega_{+}$. For the typical conditions in the present experiment, it is close to the magnetron frequency $\omega_{-}$. Hence, the ions can be regarded as weakly bound in the radial direction and consequently form a low-density structure of large radial extent. In the limit $T \rightarrow 0$, the ion number density $n$ is homogeneous and determined by the global rotation frequency $\omega_{r}$ according to (28)

$$
n=\frac{2 m \epsilon_{0}}{q^{2}} \omega_{r}\left(\omega_{c}-\omega_{r}\right)
$$

where $\omega_{c}=q B / m$ is the unperturbed cyclotron frequency. The density $n$ has a minimum for $\omega_{r}=\omega_{ \pm}$and a maximum for $\omega_{r}=\omega_{c} / 2$. The overall shape of the crystal at a given value of $\omega_{r}$ and $B$ is that of an ellipsoid of revolution with an aspect ratio $\alpha$ (axial dimension to radial dimension) determined by the trap potential $U$. For small values of $U$ (weak axial binding), it tends to be prolate (cigar-shaped along the trap axis), while for large $U$ (strong axial binding), it tends to be oblate, see the discussion in $(28)$.

\section{Experimental Setup and Procedures}

The present experiments have been performed with the SpecTrap Penning trap setup $(15,29,30)$ at the HITRAP facility (32) at GSI, Darmstadt, Germany. It uses a cylindrical, open-endcap Penning trap $(15,29,31$, 33) located in the center of a superconducting magnet for dynamic capture (34) and confinement of externally produced ions. These ions can either be obtained from a dedicated pulsed source of singly charged ions (35) or from other sources along the HITRAP low-energy beamline (36) such as an electron beam ion source (EBIS) (37) or, in the future, the HITRAP deceleration facility (32). For the present experiments, all singly charged ions were produced in the pulsed source (35) of the SpecTrap experiment, while all highly charged ions were produced in the EBIS. Both sources can be operated at the same time, and a quadrupole deflector mounted above the setup can selectively inject ions from either source into the trap. Both sources are operated to deliver roughly $10^{5}$ ions in bunches of a few $\mu$ s in duration at repetition rates of around one $\mathrm{Hz}$.

The relevant part of the low-energy beamline is shown in Fig. 1. It uses electrostatic steering, re-focussing and $90^{\circ}$ deflection to transport the ion bunches from the source of highly charged ions to the experiment, as has been detailed in (36). By a pulsed drift tube located in the horizontal part of the beamline and a subsequent pulsed drift tube inside the magnet setup, the ions can be decelerated from transport energies of 


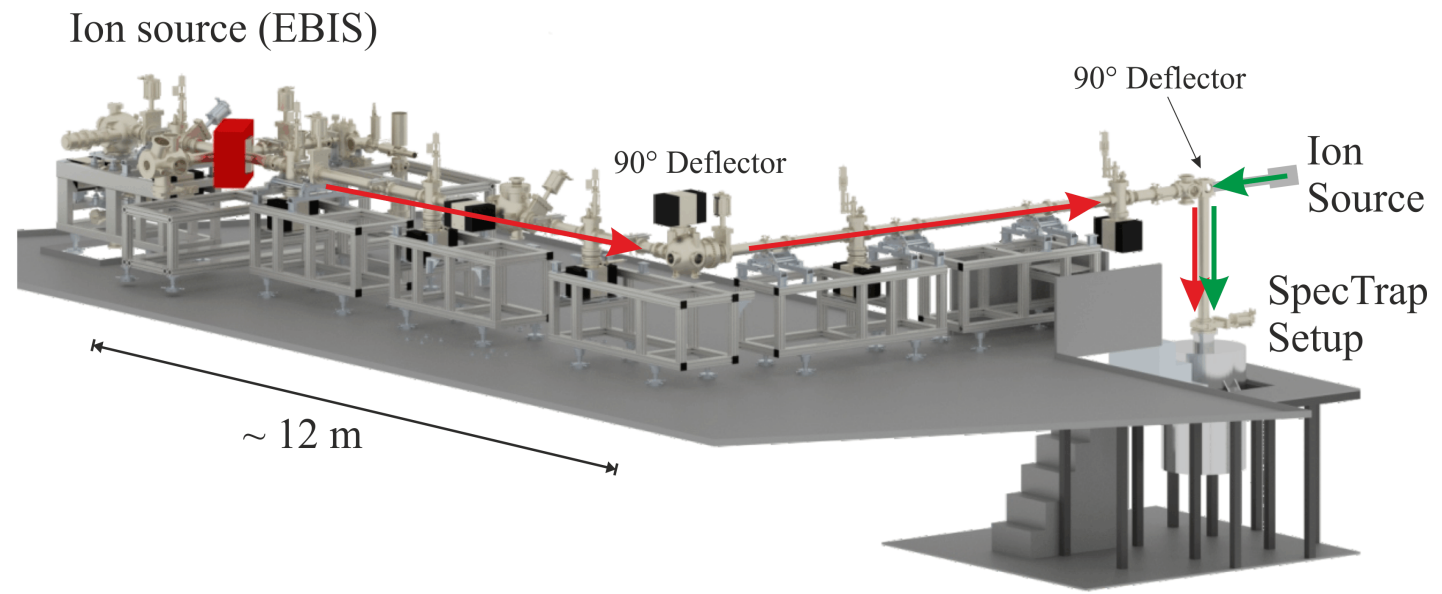

Figure 1. Schematic of the part of the HITRAP low-energy beamline connecting the EBIS offline ion source with the SpecTrap experiment. Also indicated is the beam path of highly charged ions from the EBIS (red arrows) and of singly charged ions from the pulsed source (green arrows).

up to several keV per charge to few tens of eV per charge to allow dynamic capture into the trap.

The trap is a 5-pole cylindrical open-endcap Penning trap (ring electrode, two compensation electrodes, two endcap electrodes) with an additional capture electrode on either open end, see Fig. 2. Dynamic capture is achieved by keeping the lower capture electrode on a repulsive potential, thus reflecting the ion bunch entering from above. The upper capture electrode is then pulsed from ground to the same repulsive potential, hence trapping the ions between them. This situation is depicted in Fig. 3 (a). The
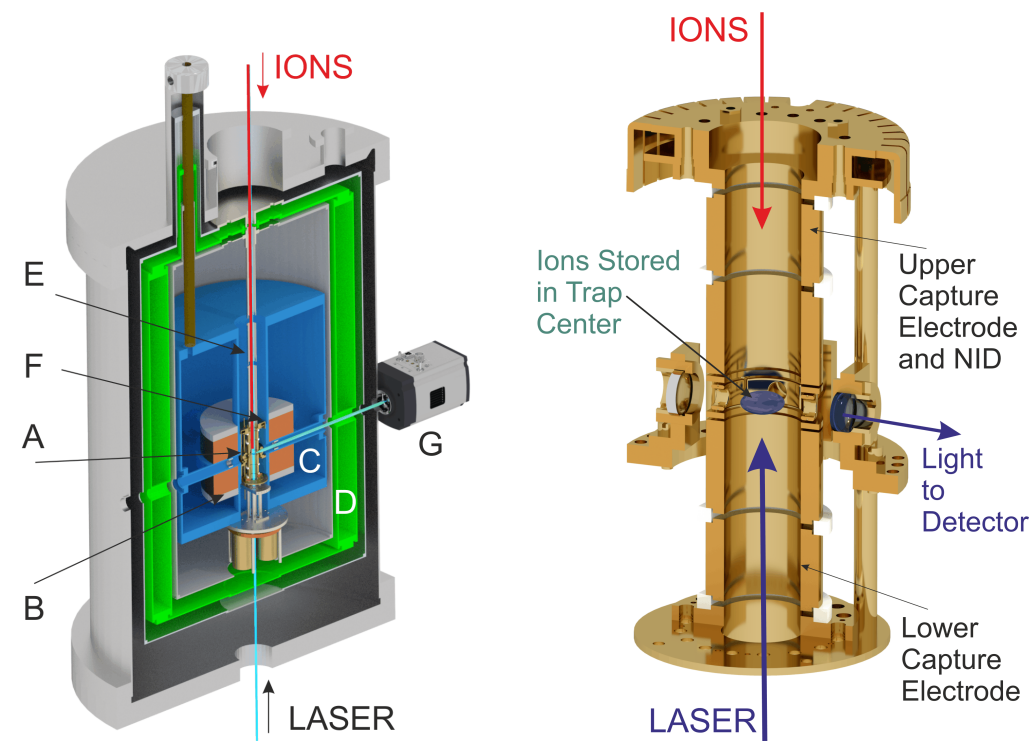

Figure 2. Left: Sectional view of the SpecTrap experimental setup with (A) Penning trap, (B) Magnet, (C) LHe dewar, (D) $\mathrm{LN}_{2}$ dewar, (E) Pulsed drift tubes, (F) Non-destructive ion detector, (G) CCD camera. Right: Sectional view of the SpecTrap Penning Trap.

upper capture electrode is equipped with a non-destructive ion detector ('NID') (38) that yields timing information on the passing ion bunch. Figure 3 (b) shows the NID signal as a function of time for a $\mathrm{Mg}^{+}$ion bunch that enters the trap, is reflected from 
the lower capture electrode, and leaves the trap again after roughly $3.5 \mu \mathrm{s}$. These ions have been injected at a kinetic energy of $365(5) \mathrm{eV}$. The information is used to optimize the capture timing. Besides the timing information, the induced signal strength gives detailed information about the number of ions entering the trap (38).

To gain further understanding of the ion dynamics upon capture, we have kept the $\mathrm{Mg}^{+}$ions confined for a variable time before opening the lower capture electrode and detecting the number of ions on a multi-channel plate (MCP) detector which can be positioned about $0.5 \mathrm{~m}$ below the trap when the laser light is not needed. The results are depicted in Fig. 3 (c). An exponential decay similar to a damped oscillator was

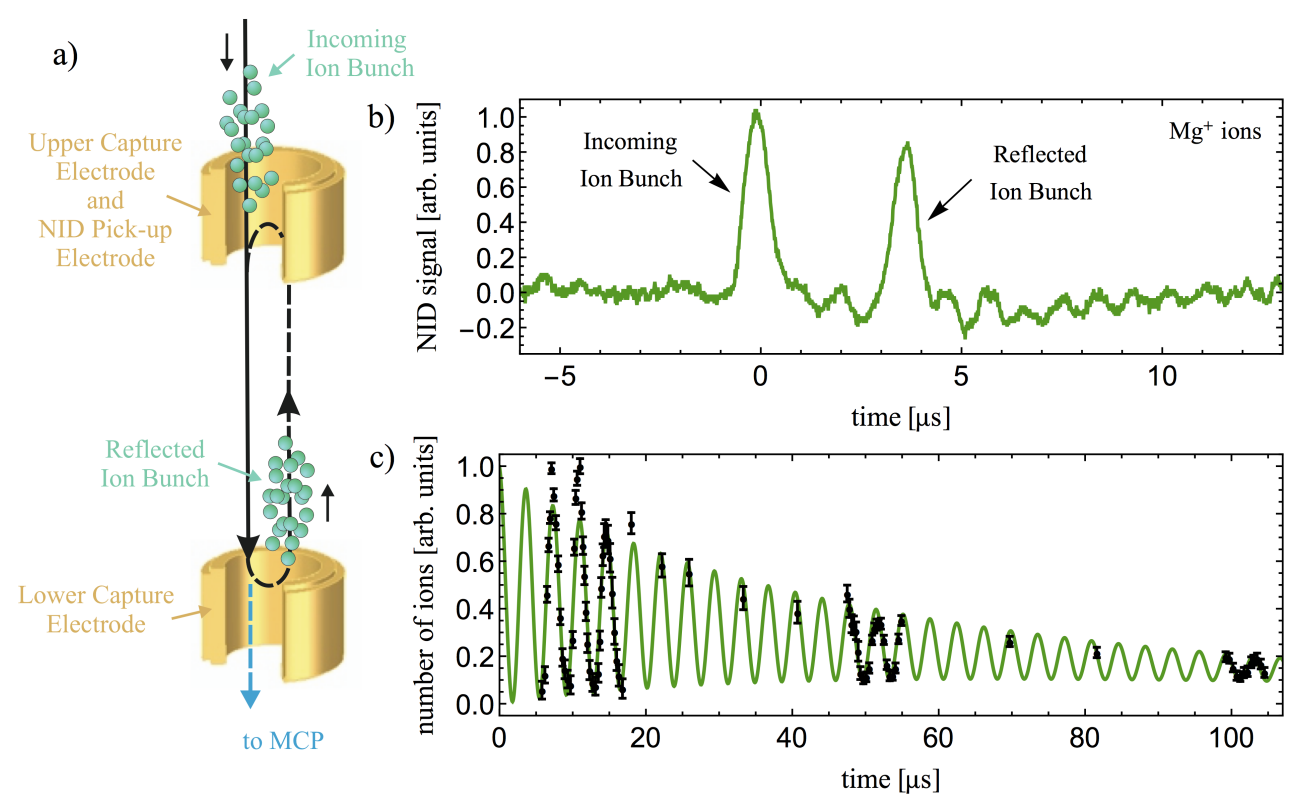

Figure 3. (a) Depiction of dynamic capture, (b) signal induced in the upper capture electrode ('NID pick-up electrode') of an ion bunch reflected from the lower capture electrode, (c) ion signal as a function of confinement time after ejection to the MCP detector.

used to fit the data. The envelope of the curve indicates the decay of the number of detected ions at the MCP due to increasing radial broadening of the ion bunch over time. The oscillation period was found to be $3.7 \mu \mathrm{s}$, whereas the decay takes place on the timescale of several tens of $\mu$ s. The sharp minima and maxima result from the conversion from axial kinetic energy to potential energy and back during the reflection process. The results show that the bunch can be seen as uniformly distributed inside the trapping region approximately $200 \mu$ s after loading. They are important for understanding the spatial distribution of the ions inside the reflection region ('reflectron') and the harmonic trap potential, if applied.

We have further investigated the dynamic capture of different pure argon-ion bunches with charge states up to $\mathrm{Ar}^{13+}$ from the HITRAP electron-beam ion source (EBIS). The parameters of this kind of ion injection are representative of the future operation with heavy highly charged ions from the HITRAP deceleration facility. Moreover, highly charged argon ions are of scientific interest for the SpecTrap experiment, as will be detailed below. This source, located about $20 \mathrm{~m}$ upstream along the low-energy ion beamline (36) provides bunches of selected ion species of up to several tens of thousands of ions at a repetition rate of several Hz. The measurement scheme for the determination of the oscillation period inside the reflectron is identical to the one used for $\mathrm{Mg}^{+}$. Table 1 shows the measured and the calculated oscillation periods 
of confined ion bunches of different charge states $q / e=4, q / e=8$ and $q / e=13$ and different kinetic energies. In all cases, the calculated values have been obtained from a numerical solution of the equation of motion of a single ion in the trap potential and are in good agreement with the experimental observations. This further corroborates the fact that the minima and maxima in the $\mathrm{Mg}^{+}$ion extraction spectra of Fig. 3 are caused by the dynamics of the oscillating ion bunch. Note that the uncertainties of the oscillation periods are much smaller than the energy uncertainties suggest, because in the given potential, the oscillation frequency is largely independent of the oscillation energy. The measurements also show that even for low mass-to-charge ratios and high

Table 1. Measured and calculated oscillation periods of captured ion bunches for different values of the ion kinetic energy per charge state and for different charge states of argon.

\begin{tabular}{|c|c|c|c|c|c|c|}
\hline Ion Species & $\mathrm{Ar}^{4+}$ & $\mathrm{Ar}^{8+}$ & $\mathrm{Ar}^{13+}$ & $\mathrm{Ar}^{13+}$ & $\mathrm{Ar}^{13+}$ & $\mathrm{Ar}^{13+}$ \\
\hline Kinetic Energy per Charge State $[\mathrm{eV}]$ & $215(10)$ & $215(10)$ & $115(10)$ & $165(10)$ & $215(10)$ & $265(10)$ \\
\hline Measured Oscillation Period $[\mu \mathrm{s}]$ & $2.968(6)$ & $2.104(2)$ & $2.097(1)$ & $1.751(1)$ & $1.625(2)$ & $1.485(1)$ \\
\hline Calculated Oscillation Period $[\mu \mathrm{s}]$ & $2.941(21)$ & $2.079(14)$ & $2.052(15)$ & $1.724(5)$ & $1.585(4)$ & $1.439(5)$ \\
\hline
\end{tabular}

ion kinetic energies, the axial oscillation period is longer than the temporal ion bunch extension of about $0.5 \mu \mathrm{s}(36)$, ensuring its complete capture. This is particularly valuable when bunches of rare ions are considered, and the loading process needs to be efficient. For the present ion species such as $\mathrm{Mg}^{+}, \mathrm{H}_{2}^{+}, \mathrm{C}^{+}, \mathrm{N}_{2}^{+}$, and $\mathrm{CO}_{2}^{+}$from our dedicated pulsed source, this is not crucial, but allows well-defined ion capture.

\section{Two-species ion crystals: Experimental Observations}

The case of single-species ion crystals in the present setup has been discussed in detail in (15), where we have achieved cooling of externally produced $\mathrm{Mg}^{+}$ions from energies of several hundreds of $\mathrm{eV}$ to energies of the crystalline state below $\mu \mathrm{eV}$ within seconds. Based on these results, two-species ion crystals have been produced by injection of singly charged admixture ions from the external ion source (on the right hand side of Fig. 1) into a previously confined cloud of laser-cooled $\mathrm{Mg}^{+}$. Besides $\mathrm{Mg}^{+}$, the external ion source can produce a variety of singly charged ion species from residual gas. Changing between the two configurations (production of $\mathrm{Mg}^{+}$and production of ions from residual gas) is achieved by adapting the electron energy used for impact ionization. A specific mass-to-charge ratio can be selectively injected into the trap by adjustment of the timing for dynamic capture. The resolving power of this process is sufficient to select between the species under discussion.

Two-species ion crystals composed of $\mathrm{Mg}^{+}(\mathrm{m} / q=24 \mathrm{u} / \mathrm{e})$ and admixture ions with mass-to-charge ratios of $m / q=2 \mathrm{u} / \mathrm{e}\left(\mathrm{H}_{2}^{+}\right), 12 \mathrm{u} / \mathrm{e}\left(\mathrm{C}^{+}\right), 28 \mathrm{u} / \mathrm{e}\left(\mathrm{N}_{2}^{+}\right)$, and $44 \mathrm{u} / \mathrm{e}\left(\mathrm{CO}_{2}^{+}\right)$ have been investigated, such that a large range of charge-to-mass ratios is covered, both below and above the value for $\mathrm{Mg}^{+}$. Laser cooling of the stored magnesium ions is achieved on the red-detuned side of the $279.55-\mathrm{nm}{ }^{2} \mathrm{~S}_{1 / 2} \rightarrow{ }^{2} \mathrm{P}_{3 / 2}$ transition with a typical detuning of half the natural linewidth of $2 \pi \times 41.8 \times 10^{6} \mathrm{~s}^{-1}$. Images of the $\mathrm{Mg}^{+}$ion fluorescence have been taken by a CCD camera along a radial optical access perpendicular to the trap axis at a rate of one frame per second. For confined ion crystals, these images show the horizontal layers of ions in the crystal. Structures in the radial plane are not resolved since the global rotation of the crystal about the trap axis is much faster than the exposure time of imaging. Details of the imaging system have been given in (15). A typical image is shown in Fig. 4 on the top right. It 
indicates the imaged confinement region as a dashed circle. The dashed vertical lines show the image area which has been used to determine the crystal layer structure from the axial intensity profile. The temporal evolution of this layer structure (as depicted in Fig. 4, top left) has been observed when admixture ions are injected into an existing $\mathrm{Mg}^{+}$ion crystal. To enhance the presentation of the layered structure, the averaged background intensity is subtracted from the image.

For admixture ion injection, the trap electrode voltages are switched as discussed above, leading to an axial displacement and rapid heating of the ion crystal accompanied by a sudden decrease of fluorescence. After a typical period of a few seconds, the crystalline structure and fluorescence are re-established. In the figures below, red crosses mark deliberate switching events without addition of ions, used as a reference for the switching events with ion injection, marked by arrows. The deliberate capture gate switching cycles without ion injection (for example at times $t$ of $20 \mathrm{~s}, 40 \mathrm{~s}, 80 \mathrm{~s}$ and $140 \mathrm{~s}$ in Fig. 4) temporarily heat the ion crystal and fully extinguish the fluorescence. In these cases, the ions re-crystallize and fluorescence fully recovers to the value extrapolated from the state before switching, i.e. there is no ion loss due to switching.

\subsection{Injection of $\mathrm{CO}_{2}^{+}$}

Images of $\mathrm{Mg}^{+}$ion crystals have been taken both before and after loading of $\mathrm{CO}_{2}^{+}$ and the evolution of the fluorescence signal and the crystal layer structures have been observed over the course of a few minutes (Fig. 4). Before loading $\mathrm{CO}_{2}^{+}(0 \leq t \leq 100 \mathrm{~s})$,

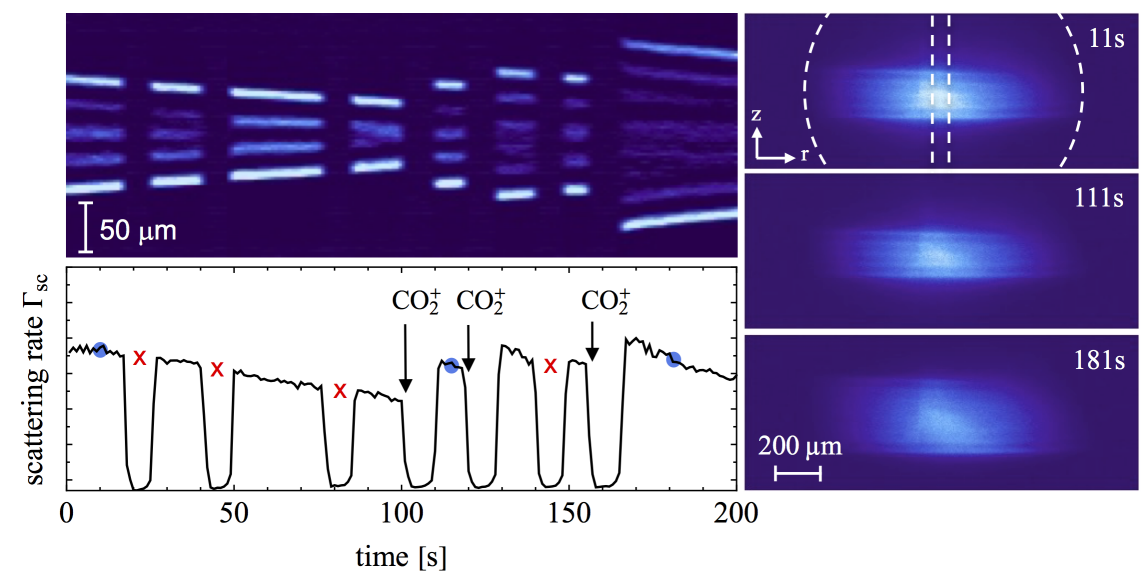

Figure 4. Right: Ion crystal images taken at the specific times indicated. Top left: enhanced presentation of the crystal layer structure as a function of time. Bottom left: fluorescence signal rate as a function of time, with injection gates for $\mathrm{CO}_{2}^{+}$indicated. The blue dots represent the times for the images on the right.

the fluorescence signal and the axial cloud extent slowly decrease. After injection of $\mathrm{CO}_{2}^{+}(m / q=44 \mathrm{u} / \mathrm{e})$, the species with the largest mass-to-charge ratio under investigation, at times $t$ of $100 \mathrm{~s}, 120 \mathrm{~s}$ and $155 \mathrm{~s}$, the axial extent of the ion cloud increases and - while the charge density, as determined from the layer distance, remains unchanged - additional crystal layers are formed. Both the total fluorescence signal in the central region of the crystal and the number of crystal layers increase, whereas the fluorescence at the center of each layer is identical before and after loading $\mathrm{CO}_{2}^{+}$. After the end of the $\mathrm{CO}_{2}^{+}$loading phase $(t \geq 170 \mathrm{~s})$, the radial transport of $\mathrm{Mg}^{+}$and the resulting decrease of the axial cloud extent continues for the $\mathrm{CO}_{2}^{+}-\mathrm{Mg}^{+}$crystal with the same rate as for the single-species $\mathrm{Mg}^{+}$ion cloud. 


\subsection{Injection of $N_{2}^{+}$}

Comparable effects have been observed after injection of $\mathrm{N}_{2}^{+}$ions $(m / q=28 \mathrm{u} / \mathrm{e})$, see Fig. 5. The first injection of $\mathrm{N}_{2}^{+}$at $t=10 \mathrm{~s}$ is accidentally inefficient. Similar to the

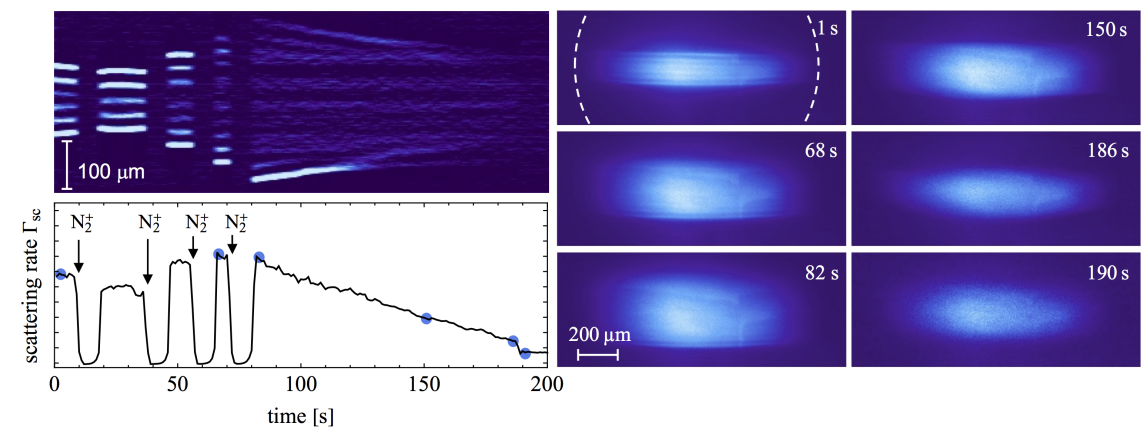

Figure 5. Right: Ion crystal images taken at the specific times indicated. Top left: crystal layer structure as a function of time. Bottom left: fluorescence signal rate as a function of time, with injection gates for $\mathrm{N}_{2}^{+}$ indicated.

non-loading capture gate switching event at $t=40 \mathrm{~s}$ in Fig. 4, fluorescence recovers at a level matching the reduction in the number of layers (6 to 5 layers) as a consequence of the standard ion number decay due to loss. Thereafter, the cloud size increases upon further loading of $\mathrm{N}_{2}^{+}$at $t=35 \mathrm{~s}, t=55 \mathrm{~s}$, and $t=70 \mathrm{~s}$ and the density is constant before and after each injection of $\mathrm{N}_{2}^{+}$. The crystal structure of the two-species ion cloud is clearly observable. The fluorescence signal for a specific layer is again found to be approximately unaltered before and after loading of $\mathrm{N}_{2}^{+}$and consequently the total fluorescence increases as additional layers are formed. After the last injection of $\mathrm{N}_{2}^{+}$(at $t=70 \mathrm{~s}$ ), the fluorescence rate and the axial cloud extent slowly decrease due to gradual ion loss, until at $t=185 \mathrm{~s}$ a sudden drop of fluorescence occurs, which we attribute to heating effects not balanced by laser cooling giving a new equilibrium with higher temperature and lower fluorescence for our laser detuning.

\subsection{Injection of $C^{+}$}

Figure 6 shows a typical injection process of $\mathrm{C}^{+}(\mathrm{m} / q=12 \mathrm{u} / \mathrm{e})$. Again, the crystal

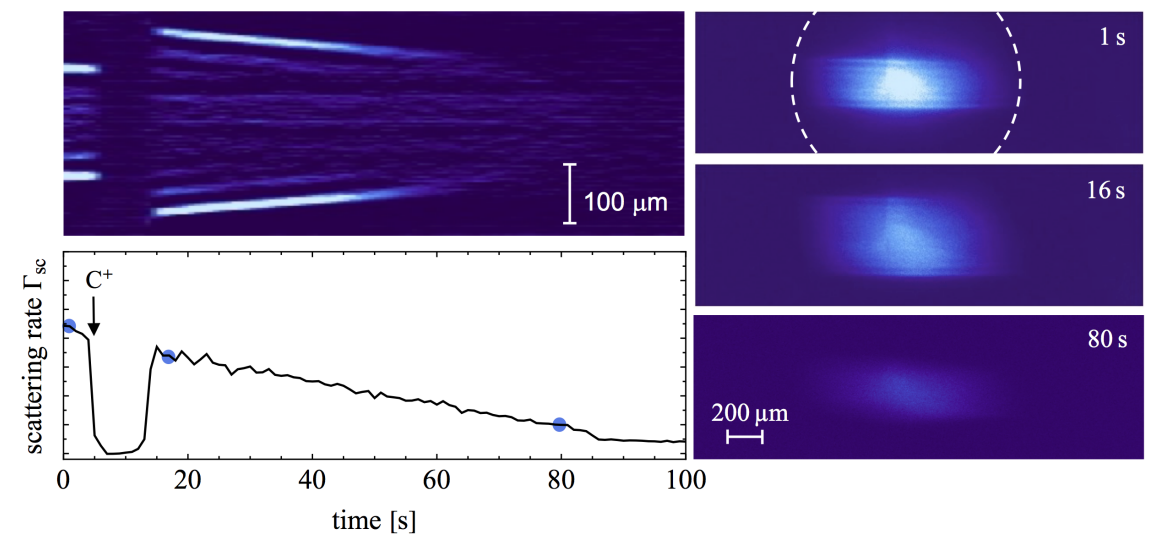

Figure 6. Right: Ion crystal images taken at the specific times indicated. Top left: crystal layer structure as a function of time. Bottom left: fluorescence signal rate as a function of time, with injection gate for $\mathrm{C}^{+}$ indicated. 
structure is re-established after injection of $\mathrm{C}^{+}$and again the axial cloud extension increased due to the formation of additional crystal layers. However, in contrast to the cases above, no increase of the total fluorescence following the injection of $\mathrm{C}^{+}$is observed (Fig. 6, bottom left). Since the cloud extension increases nonetheless, this means that the average fluorescence per layer is reduced. With a constant charge density per layer, this must be caused by the $\mathrm{C}^{+}$ions being incorporated close to the crystal axis as expected, see section 5.2. Similar to the observations with $\mathrm{N}_{2}^{+}$, the fluorescence rate and the axial cloud extent decrease after re-establishment of the crystal structure at $t=16 \mathrm{~s}$, until at $t=85 \mathrm{~s}$ a sudden drop of fluorescence occurs, similar to the one discussed in the previous section.

\subsection{Injection of $\mathrm{H}_{2}^{+}$}

The lightest ion species under investigation was $\mathrm{H}_{2}^{+}(\mathrm{m} / q=2 \mathrm{u} / \mathrm{e})$. Immediately after injection of $\mathrm{H}_{2}^{+}$, fluorescence is fully lost as in the previous cases. In contrast to these, the fluorescence recovers only to a fraction of the initial value and the images show no crystalline structure, whereas the axial cloud extension remains constant (Fig. 7). This
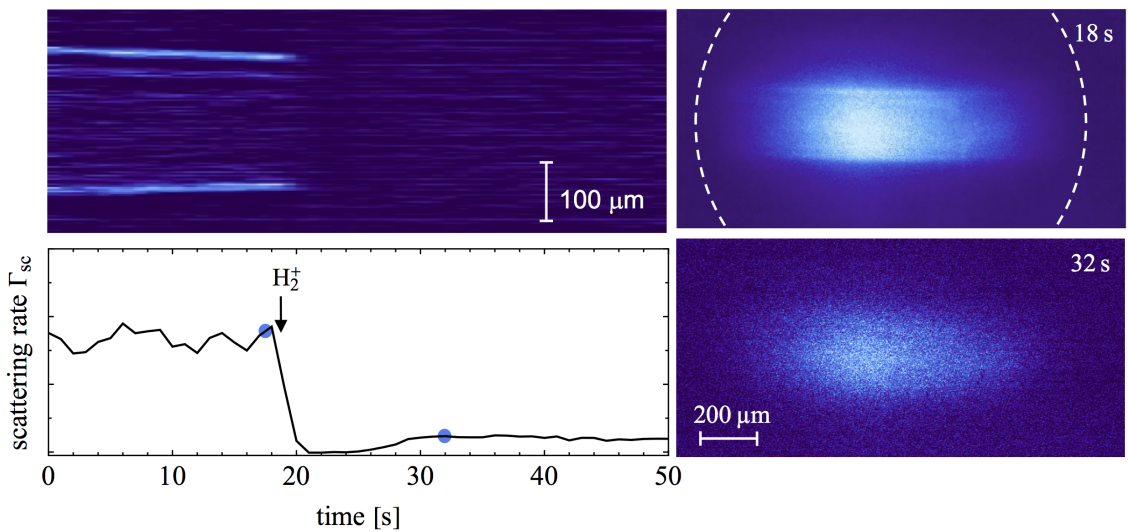

Figure 7. Right: Ion crystal images taken at the specific times indicated. Top left: crystal layer structure as a function of time. Bottom left: fluorescence signal rate as a function of time, with injection gate for $\mathrm{H}_{2}^{+}$ indicated.

indicates that no particles are lost in the capture process and the decreased signal rate is a genuine characteristic of the $\mathrm{H}_{2}^{+}-\mathrm{Mg}^{+}$-ion cloud.

\section{Discussion}

\subsection{Involved Processes}

We briefly discuss the relevant processes which determine the ion dynamics. Under the present experimental conditions, the confined $\mathrm{Mg}^{+}$ion crystals are subjected to laser cooling and to heating by interaction with residual gas (mainly helium at a pressure of the order of $10^{-8}$ mbar and at the ambient temperature of about $4 \mathrm{~K}$ ), with laser cooling dominating by far, hence the rapid crystallization previously observed for pure $\mathrm{Mg}^{+}$crystals (15). Note, that for $\mathrm{Mg}^{+}$at initially high energies (far above $4 \mathrm{~K}$ ), the residual gas provides efficient buffer gas cooling, as discussed in (15), whereas for cold $\mathrm{Mg}^{+}$in the laser-cooling regime and close to the Doppler limit (far below $4 \mathrm{~K}$ ) it acts as a heating mechanism. Energy dissipation from the magnetron motion for example 
by buffer gas cooling gas tends to increase the radius of the magnetron motion (16), and may eventually lead to ion loss from the confinement region.

The admixture ions are subjected to sympathetic cooling via the laser-cooled $\mathrm{Mg}^{+}$ as will be detailed below and to similar heating by residual gas. As sympathetic cooling depends on more parameters than laser cooling which vary during the course of observation, one can expect the competition between these two processes to display more features.

In all cases under consideration, there is quasi-continuous ion loss on long time scales as would be expected in the presence of residual gas. The observed time constants agree with the expected value of about $100 \mathrm{~s}$ for loss due to magnetron expansion of the ion motion beyond the confinement region $(40)$. This loss cannot directly be deduced from the total observed amount of fluorescence since the light collection region is finite, and there is centrifugal separation between different ion species, as will be detailed in the next section.

\subsection{Centrifugal Separation}

The different observed characteristics of the present two-species ion crystals can be classified with regard to the mass-to-charge ratio of the sympathetically cooled species. Generally, for a multi-species plasma at low temperature, its rotation tends to produce centrifugal separation of its components. As long as the plasma is not yet in thermal equilibrium, the different species will rotate with different frequencies, and collisions between the two species will cause a net transfer of momentum. As a consequence, an inward radial flux of one species and an outward radial flux of the other species is created (41). The plasma evolves until it reaches thermal equilibrium, which is characterized by a rigid-body rotation. In this case, both species rotate with the same frequency $\omega_{r}$ but experience a different centrifugal potential due to their different masses. The degree of separation depends on the plasma temperature and on the difference of the involved mass-to-charge ratios. If the separation is complete, the species are arranged in separate concentric rings with the ion with smaller mass-tocharge ratio in the center of the trap. Such complete separation has been observed in several experiments, for example for two-species clouds consisting of cold beryllium and mercury ions (43) as well as for combinations of singly charged and highly charged ions (42).

The increasing observed fluorescence rates after injection of ions with masses larger than $\mathrm{Mg}^{+}$, as shown in Figs. 4 and 5, can be attributed to radial separation of the different ion species. For $\mathrm{CO}_{2}^{+}$and $\mathrm{N}_{2}^{+}$, the mass-dependence of the centrifugal potential results in an accumulation of the fluorescing $\mathrm{Mg}^{+}$ions near the trap center, whereas the heavier ions are pushed to larger radii outside the observation region. The increase in total ion number through injection causes the formation of additional crystal layers. As a consequence, the fluorescence from the central area signal increases since these layers have the same $\mathrm{Mg}^{+}$fluorescence density as the single-species ion crystal.

For ion species with masses smaller than $\mathrm{Mg}^{+}$(Figs. 6 and 7), the non-fluorescing ions accumulate near the trap center and the $\mathrm{Mg}^{+}$ions are pushed out of the central region. Although the number of visible layers increased upon $\mathrm{C}^{+}$injection, the total fluorescence decreased because the detected fluorescence now stems from the less intense wings of the laser field distribution. The same effect can be observed for $\mathrm{H}_{2}^{+}$. Here, the fluorescence decreases even more than for $\mathrm{C}^{+}$, which can be attributed to a more complete separation of the two ion species because of their larger mass difference 
and heating effects, as discussed below.

One unresolved feature is that in such a situation, one would expect a central depression in the radial fluorescence distribution due to the displacement of the $\mathrm{Mg}^{+}$ ions. In experiments where the complete cloud was imaged, such structures were reported (42). In the results presented here, no such effect was observed. This may be a misalignment artefact when the center of the imaged area is not identical to the center of the trap. There could also be the influence of the integration along the line of sight as well as the inhomogeneous illumination with a Gaussian profile laser beam that could cause the central depression to be overcompensated. The observation could also be due to incomplete separation of the species due to the raised temperature in this case.

\subsection{Temperatures}

As our radial projections of the magnesium ion cloud can reveal ordered structures only along the axial direction and not within each layer, we give limits on the actual $\mathrm{Mg}^{+}$ion temperature $T$ before injection of the second species in the range of $T_{D}=$ $1 \mathrm{mK}<T<6 \mathrm{mK}=T_{\Gamma}$, where $T_{D}$ is the Doppler-limited temperature $(15)$ and $T_{\Gamma}$ is the temperature corresponding to a value of $\Gamma_{p}=140$ at the ion number density $n=3.4 \times 10^{7} \mathrm{~cm}^{-3}$. This is in correspondence with detailed simulations of ion Coulomb crystals which show that as $\Gamma_{p}$ approaches a value of about 140, the ion cloud shows distinct density modulations along the axial direction as observed (with only a small amount of diffusion of ions from one layer to another), whereas in the radial direction the ions are more likely to change places (a liquid-like state) (44). This limit of the ion temperature is corroborated by our recent investigations of the cooling behaviour of singly charged magnesium ions (15), which yield an upper limit for the ion temperature of about $10 \mathrm{mK}$. Here, the ion temperature was extracted from the Doppler-broadend lineshape of the optical transition upon laser cooling.

After injection and sympathetic cooling of admixture ions with mass-to-charge ratios larger than that of magnesium, the crystalline structure is fully restored, see Figs. 4 and 5. This indicates that the magnesium ion temperature is still below $10 \mathrm{mK}$. As the crystalline state is preserved while the admixture ions are present, strong heating directly after loading introduced by this second ion species can be ruled out. Since the ions equilibrate by their Coulomb interaction, one may assume the second species to adopt a similar temperature, given sufficiently long interaction times. We hence assume $10 \mathrm{mK}$ as the upper limit for the temperature of all ions in the crystal.

Upon loading of admixture ions, the observed long-term decrease in total fluorescence is faster than explicable by particle loss from the trap alone. Such a decrease is not observed for single-species $\mathrm{Mg}^{+}$crystals (15), and indicates some amount of heating introduced by the admixture ions which adds to the residual gas heating. In the cases of $\mathrm{N}_{2}^{+}$and $\mathrm{C}^{+}$, after a few tens of seconds it leads to the crystal structure of the cloud no longer being observable.

There is also an additional, abrupt decrease of fluorescence seen at $t=85 \mathrm{~s}$ for $\mathrm{C}^{+}$and at $t=185 \mathrm{~s}$ for $\mathrm{N}_{2}^{+}$. Since the axial cloud extension before and after this step in fluorescence is similar, also this effect is not attributed to ion loss. It may instead be attributed to heating by residual gas overcoming sympathetic cooling in these specific circumstances. The vanishing of the crystal structure is then either due to insufficient signal-to-noise ratio, or because the ion cloud transits into a liquid-like state. The results for $\mathrm{H}_{2}^{+}$suggest that the transition to this state occurs immediately 
after loading without a crystalline structure being established at all.

\section{Model of Sympathetic cooling}

The above measurements prove sympathetic cooling of a second ion species by the laser-cooled $\mathrm{Mg}^{+}$ions. However, they do not give access to the precise time scale of sympathetic cooling. This is partly due to the influence of the residual gas, but more so because the cooling time constants are expected to be smaller than presently observable within our video frame resolution of about one second. In sympathetic cooling as it is discussed here, the admixture ions equilibrate with the laser-cooled ions via the Coulomb interaction. For full spatial overlap of the two ion ensembles, and assuming that the coolant is not heated significantly by the admixed species, the sympathetic cooling time constant $\tau$ can be estimated by (39)

$$
\tau \approx\left(4 \pi \epsilon_{0}\right)^{2} \frac{m m_{A}}{q^{2} q_{A}^{2}} \frac{1}{n \ln \Lambda}\left(\frac{k_{B} T}{m}+\frac{k_{B} T_{A}}{m_{A}}\right)^{3 / 2},
$$

where quantities with no index refer to the coolant ions (presently $\mathrm{Mg}^{+}$) and the index ' $\mathrm{A}$ ' refers to the admixture ions to be cooled. The temperature evolution of the species to be cooled is then given by $\partial T_{A} / \partial t=-\left(T_{A}-T\right) / \tau$. Note, that the time constant of equilibration $\tau$ is proportional to the inverse square of the ion charges, i.e. sympathetic cooling is particularly efficient for high charge states. The parameter $\ln \Lambda$ is the so-called 'Coulomb logarithm' which is described in detail in (39) and for the present conditions is a number close to 15 . From the CCD images, a typical

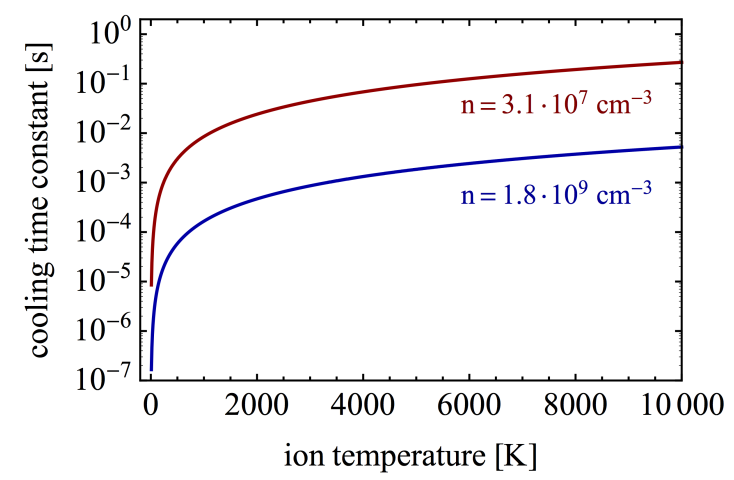

Figure 8. Sympathetic cooling time constant $\tau$ of a $\mathrm{CO}_{2}^{+}$ion as a function of initial ion temperature for the minimum (in brown) and maximum (in blue) coolant ion number density under the present experimental conditions.

magnesium ion-ion distance is found to be approximately $20 \mu \mathrm{m}$, corresponding to a density of $n \approx 3.4 \times 10^{7} / \mathrm{cm}^{3}$. Assuming ions such as $\mathrm{CO}_{2}^{+}$to be cooled from an initial temperature of $T_{A} \approx 10^{4} \mathrm{~K}$ (corresponding to about one $\mathrm{eV}$ ), the cooling time constant $\tau$ is below one second and gets even smaller for smaller initial temperatures, see Fig. 8. For ions of present interest such as ${ }^{40} \mathrm{Ar}^{13+}(46)$ under the same conditions, the cooling time constant is of the order of milliseconds and below. This species is of particular interest for commissioning of the system with highly charged ions, and for a precision measurement of the ${ }^{2} \mathrm{P}_{3 / 2}$ to ${ }^{2} \mathrm{P}_{1 / 2}$ transition energy and lifetime. For injected ions, the spatial ion overlap in the confinement region is of course initially smaller than assumed here, and correspondingly, sympathetic cooling is somewhat 
slower. Also, centrifugal separation may reduce the sympathetic cooling efficiency at later times. To quantify this properly requires a detailed account of ion trajectories which is beyond our scope, but in any case, we deduce the cooling to be unresolved at the temporal resolution of our observation. On the positive side, this means that even for singly charged ions, and even more so for higher charge states, sympathetic cooling is fast relative to the time scale of ion confinement in cryogenic trap experiments such as the present one.

\section{Conclusion}

Complementing our previous studies of single-species ion Coulomb crystals in the SpecTrap Penning trap (15), we have investigated two-species ion crystals formed by sympathetic cooling of a second ion species injected into previously confined and lasercooled $\mathrm{Mg}^{+}$. For this, we have imaged the temporal evolution of ion crystal structures of mesoscopic magnesium ion crystals injected with singly charged admixture ions of mass-to-charge ratios $m / q=2 \mathrm{u} / \mathrm{e}\left(\mathrm{H}_{2}^{+}\right), m / q=12 \mathrm{u} / \mathrm{e}\left(\mathrm{C}^{+}\right), m / q=28 \mathrm{u} / \mathrm{e}\left(\mathrm{N}_{2}^{+}\right)$, and $m / q=44 \mathrm{u} / \mathrm{e}\left(\mathrm{CO}_{2}^{+}\right)$. Sympathetic cooling of admixture ions was observed and the results are in agreement with expectations from theory and previous experiments on centrifugal separation of ion species in a Penning trap. For all admixtures except $\mathrm{H}_{2}^{+}$an ion Coulomb crystal was established in the mixture, giving an upper temperature limit of about $10 \mathrm{mK}$ for all ion components. Such sympathetic cooling is favourable when ions without suitable transitions for laser cooling are to be cooled significantly below ambient temperature, for example for precision optical spectroscopy. The presented method and results are valuable for precision spectroscopy of highly charged ions such as for example $\mathrm{Ar}^{13+}$ and ions of higher charge states as foreseen in the SpecTrap experiment $(47)$. Also, the methods described in this paper can be used to produce large and cold samples of heavy diatomic molecular cations such as $\mathrm{Ni}_{2}^{+}, \mathrm{Mn}_{2}^{+}$or $\mathrm{Co}_{2}^{+}$ by means of sympathetic cooling. With ion numbers of up to $10^{5}$ and ion temperatures below $1 \mathrm{~K}$, these molecular ion Coulomb crystals represent ideal candidates for x-ray magnetic circular dichroism spectroscopy (48), in order to reveal the ground state properties of such homonuclear metal ions.

\section{Acknowledgement(s)}

We acknowledge the many contributions of the late Danny Segal to this project over many years. We also acknowledge the work of all members of the SpecTrap collaboration. We thank Bernhard Maaß, Alexander Martin, Oliver Kaleja, Jörg Krämer, Tim Ratajczyk and Rodolfo Sanchez for their support in the execution of the experiment. We gratefully acknowledge support by the BMBF under contract 05P15RDFAA, HIC for FAIR within the LOEWE program by the federal state Hessen, the DFG under contract BI 647/5-1 and the EPSRC under grant number EP/D068509/1. S.S. and T.M. have contributed equally to this work. S.S. and T.M. acknowledge support from HGSHIRe. The experiments have been performed within the framework of the HITRAP facility at GSI and FAIR, Darmstadt. 


\section{References}

(1) W. Demtröder, Laser Spectroscopy, Springer, Heidelberg (2003)

(2) G. Werth, V.N. Gheorghe and F.G. Major, Charged Particle Traps, Springer, Heidelberg (2005)

(3) P. Ghosh, Ion Traps, Oxford University Press, Oxford (1995)

(4) W.M. Itano, J.C. Bergquist, J.J. Bollinger and D.J. Wineland, Physica Scripta T59 (1995) 106-120

(5) J.F. Goodwin, G. Stutter, R.C. Thompson, and D.M. Segal, Phys. Rev. Lett. 116 (2016) $143002(5)$

(6) G. Savard, St. Becker, G. Bollen, H.-J. Kluge, R.B. Moore, Th. Otto, L. Schweikhard, H. Stolzenberg, and U. Wiess, Physics Letters A 158 (1991) 247-252

(7) H. G. Dehmelt, Adv. At. Mol. Phys. 5 (1969) 109-154

(8) M. Vogel, H. Häffner, K. Hermanspahn, S. Stahl, J. Steinmann, and W. Quint, Phys. Rev. A 90 (2014) 043412(10)

(9) W. M. Itano and D. J. Wineland, Phys. Rev. A 25 (1982) 35-54

(10) J. Eschner, G. Morigi, F. Schmidt-Kaler, and R. Blatt, J. Opt. Soc. Am. B 20 (2003) $1003-1015$

(11) H.J. Metcalf und P. Straten, Laser cooling and trapping of neutral atoms, Wiley Online Library (2007)

(12) P. van der Straten and H. Metcalf, Atoms and Molecules Interacting with Light Cambridge University Press (2016)

(13) M. Vogel and W. Quint, Phys. Rep. 490 (2010) 1-47

(14) M.A. van Eijkelenborg, M.E.M. Storkey, D.M. Segal, and R.C. Thompson, Phys. Rev. A 60 (1999) 3903-3910

(15) T. Murböck, S. Schmidt, G. Birkl, W. Nörtershäuser, R.C. Thompson, and M. Vogel, Phys. Rev. A 94 (2016) 043410(10)

(16) L S. Brown and G. Gabrielse, Rev. Mod. Phys. 58 (1986) 233-311

(17) D.H.E. Dubin and T.M. ONeil, Rev. Mod. Phys. 71 (1999) 87-172

(18) R.C. Thompson, Contemporary Physics 56 (2015) 63-79

(19) F. Diedrich, E. Peik, J.M. Chen, W. Quint, and H. Walther, Phys. Rev. Lett. 59 (1987) 2931-2934

(20) G. Birkl, S. Kassner and H. Walther, Nature 357 (1992) 310-313

(21) I. Waki, S. Kassner, G. Birkl, and H. Walther, Phys. Rev. Lett. 68 (1992) 2007-2010

(22) T.B. Mitchell, J.J. Bollinger, D.H.E. Dubin, X.-P. Huang, W.M. Itano, and R.H. Baughman, Science 282 (1998) 1290-1293

(23) M. Drewsen, C. Brodersen, L. Hornekr, J. S. Hangst, and J. P. Schifffer, Phys. Rev. Lett. 81 (1998) 2878-2881

(24) L. Hornekaer and M. Drewsen, Phys. Rev. A 66 (2002) 013412(12)

(25) B.C. Sawyer, J.W. Britton, A.C. Keith, C.-C.J. Wang, J.K. Freericks, H. Uys, M.J. Biercuk, and J.J. Bollinger, Phys. Rev. Lett. 108 (2012) 213003(5)

(26) S. Mavadia, J.F. Goodwin, G. Stutter, S. Bharadia, D.R. Crick, D.M. Segal, and R.C. Thompson, Nature Communications 4 (2013) 2571(7)

(27) S. Bharadia, M. Vogel, D.M. Segal and R.C. Thompson, Appl. Phys. B 107 (2012) 11051115

(28) J.J. Bollinger, D.J. Heinzen, F.L. Moore, W.M. Itano, and D.J. Wineland, Phys Rev. A 48 (1993) 525-545

(29) Z. Andelkovic, R. Cazan, W. Nörtershuser, S. Bharadia, D.M. Segal, R.C. Thompson, R. Jöhren, J. Vollbrecht, V. Hannen, and M. Vogel, Phys. Rev. A 87 (2013) 033423(9)

(30) M. Vogel, D.F.A. Winters, D.M. Segal, and R.C. Thompson, Rev. Sci. Inst. 76 (2005) $103102(7)$

(31) S. Schmidt, C. Geppert, and Z. Andelkovic, Hyp. Int. 227 (2014) 29-43

(32) H.-J. Kluge, T. Beier, K. Blaum, L. Dahl, S. Eliseev, F. Herfurth, B. Hofmann, O. Kester, S. Koszudowski, C. Kozhuharov, G. Maero, W. Nörtershäuser, J. Pfister, W. Quint, U. 
Ratzinger, A. Schempp, R. Schuch, T. Stöhlker, R.C. Thompson, M. Vogel, G. Vorobjev, D.F.A. Winters, and G. Werth, Advances in Quantum Chemistry 53 (2008) 83-90

(33) G. Gabrielse, L. Haarsma and S.L. Rolston, Int. J. Mass Spectr. Ion Proc. 88 (1989) 319-332

(34) H. Schnatz, G. Bollen, P. Dabkiewicz, P. Egelhof, F. Kern, H. Kalinowsky, L. Schweikhard, and H. Stolzenberg, Nucl. Inst. Meth. A 251 (1986) 17-20

(35) T. Murböck, S. Schmidt, Z. Andelkovic, G. Birkl, W. Nörtershäuser, and M. Vogel, Rev. Sci. Inst. 87 (2016) 043302(5)

(36) Z. Andelkovic, F. Herfurth, N. Kotovskiy, K. König, B. Maaß, T. Murböck, D. Neidherr, S. Schmidt, J. Steinmann, M. Vogel, and G. Vorobjev, Nucl. Inst. Meth. A 795 (2015) 109-114

(37) A. Sokolov, F. Herfurth, O. Kester, Th. Stoehlker, A. Thorn, G. Vorobjev and G. Zschornack, Journal of Instrumentation 5 (2010) C11001(9)

(38) S. Schmidt, T. Murböck, Z. Andelkovic, G. Birkl, W. Nörtershäuser, S. Stahl, and M. Vogel, Rev. Sci. Inst. 86 (2015) 113302(6)

(39) J. D. Huba, NRL Plasma Formulary, Beam Physics Branch, Plasma Physics Division, Naval Research Laboratory, Washington DC (2013)

(40) S. Schmidt, Untersuchung mesoskopischer Coulomb-Kristalle zum sympathetischen Kühlen hochgeladener Ionen und direkte Bestimmung der Massendifferenz von 123Te und 123Sb, PhD thesis, Darmstadt (2016)

(41) T.M. O'Neil, Phys. Fluids 24 (1981) 1447-1451

(42) L. Gruber, J.P. Holder and D. Schneider, Physica Scripta 71 (2005) 60-107

(43) D.J. Larson, J.C. Bergquist, J.J. Bollinger, W.M. Itano, and D.J. Wineland, Phys. Rev. Lett. 57 (1986) 70-73

(44) D.H.E. Dubin and T.M. O'Neil, Phys. Rev. Lett. 60 (1988) 511-514

(45) M. Vogel and W. Quint, New Journal of Physics 11 (2009) 013024(12)

(46) D. von Lindenfels, M. Wiesel, D.A. Glazov, A.V. Volotka, M.M. Sokolov, V.M. Shabaev, G. Plunien, W. Quint, G. Birkl, A. Martin, and M. Vogel, Phys. Rev. A 87 (2013) $023412(10)$

(47) S. Albrecht, S. Altenburg, C. Siegel, N. Herschbach and G. Birkl, Appl. Phys. B 107 (2012) 1069-1074

(48) V. Zamudio-Bayer, R. Lindblad, C. Bülow, G. Leistner, A. Terasaki, B.v. Issendorff, and J.T. Lau, J. Chem. Phys. 145 (2016) 194302(5) 